Artículo

\title{
Tasa de intercambio neto de bióxido de carbono de un viñedo durante el ciclo de crecimiento
}

\author{
Alejandro Zermeño-González ${ }^{1 \S}$ \\ Addy Patricia Bravo-Escalante ${ }^{1}$ \\ Santos Gabriel Campos-Magaña ${ }^{2}$ \\ Homero Ramírez-Rodríguez ${ }^{3}$ \\ Jorge Méndez-González ${ }^{4}$
}

${ }^{1}$ Departamento de Riego y Drenaje. ${ }^{2}$ Departamento de Maquinaria Agrícola. ${ }^{3}$ Departamento de Horticultura. ${ }^{4}$ Departamento Forestal-Universidad Autónoma Agraria Antonio Narro. Calzada Antonio Narro núm. 1923, Col. Buenavista, Saltillo, Coahuila, México.

${ }^{\S}$ Autor para correspondencia: azermenog@ hotmail.com.

\section{Resumen}

Además de la cosecha de uva para consumo en fresco, elaboración de jugos y la producción de vinos, los viñedos (Vitis vinifera L.) por su condición de plantas leñosas y longevas pueden tener una participación importante en la asimilación y retención del carbono atmosférico. El objetivo de este estudio fue evaluar la tasa de intercambio neto de bióxido de carbono del ecosistema por sus siglas en inglés (NEE) en un viñedo durante su ciclo de producción, y su relación con el secuestro de carbono atmosférico. El estudio se realizó (de abril a diciembre de 2018) en un viñedo del cultivar Shiraz de 11 años, de la Vinícola San Lorenzo, Parras, Coahuila. La tasa de flujo de bióxido de carbono entre el dosel del viñedo y la atmósfera, a través de los meses de crecimiento se midió con los sensores de un sistema de covarianza eddy. De abril a noviembre el viñedo actúa como un sumidero de carbono atmosférico y durante mayo, junio y julio se tuvieron los valores mayores de $\mathrm{NEE}$, con un valor promedio de $-3.014 \mathrm{~g} \mathrm{C} \mathrm{m}^{-2} \mathrm{~s}^{-1}$. El carbono almacenado en la madera de las plantas del viñedo fue $3.35 \mathrm{t} \mathrm{C} \mathrm{ha}^{-1}$. Estos resultados muestran que, los viñedos son sistemas agrícolas que pueden tener una participación importante en la mitigación del bióxido de carbono atmosférico, que aunado a su condición de plantas leñosas-longevas y las grandes superficies establecidas de viñedos en México y el mundo, son ecosistemas de almacenamientos de carbono muy importantes.

Palabras clave: Vitis vinifera L., fotosíntesis, radiación fotosintéticamente activa, secuestro de carbono.

Recibido: marzo de 2021

Aceptado: junio de 2021 


\section{Introducción}

La vid (Vitis vinífera L.) es una planta trepadora, leñosa cuyo fruto es la uva con la que se elaboran los vinos, es originaria de Asia y es conocida desde la prehistoria. Los españoles introdujeron este cultivo a América del Norte (Vinetur, 2017). En México, la siembra de vid para la producción de vino inicio en 1597 en el Valle de Parras en la Hacienda San Lorenzo (CMV, 2018). Actualmente, la producción de uva para vino asciende a 6474 ha, donde Baja California concentra el mayor porcentaje de producción con 57\% del total de los 11 estados productores (SADER, 2018). En 2019, la superficie mundial global de viñedos, incluyendo todas las orientaciones productivas fue 7.402 millones de hectáreas. España tiene la superficie mayor (966 000 ha), seguida de China y Francia con 855000 y 749000 ha (OIV, 2019).

En México, los viñedos agrupan 2900 productores, que generan más de 3000 empleos de manera directa e indirecta y participan más de 500 mil jornaleros agrícolas en apoyo a las actividades de poda y cosecha (El financiero, 2018). Además de la importancia económica y social de la vid, los viñedos participan en la asimilación y retención del carbono atmosférico, ya que son plantas leñosas y longevas que puede estar en producción por más de 40 años.

La vid tiene una vida media de 50 años, aunque algunas viñas pueden vivir hasta 100 años (Cano, 2015; Domínguez de la Iglesia; 2018). La tasa de intercambio neto de bióxido de carbono (NEE) representa la capacidad de asimilación de $\mathrm{CO}_{2}$ de una superficie vegetal, que depende del tipo de vegetación, estado de crecimiento, condiciones climáticas y humedad del suelo.

Diversos estudios han reportado NEE de diferentes tipos de bosques y localidades (Carrara et al., 2003; Desai et al., 2005; Yi et al., 2008). En diferentes huertas de frutales (Testi et al., 2008; Martin-Gorriz et al., 2011; Zanotelli et al., 2013). Y en diferentes variedades de viñedos (Nardino, et al., 2007; Smart et al., 2009; Vendrame, 2016). Los valores integrados diarios, mensuales o por ciclo de crecimiento del NEE $\left(\mathrm{mol} \mathrm{m}^{-2}\right)$, pueden indicar la capacidad de asimilación del bióxido de carbono atmosférico de un determinado ecosistema vegetal. Estudios previos han evaluado la tasa de fijación de bióxido de carbono de viñedos de diferentes cultivares y varias edades (Guo et al., 2014; Pitacco y Meggio, 2016).

El carbón asimilado y que se integra al crecimiento de la madera se define como secuestro de carbono. Que depende del tipo de ecosistema vegetal, condiciones climáticas y humedad del suelo. Por su longevidad y el volumen de biomasa acumulada, los bosques en condiciones climáticas favorables tienen un alto potencial de secuestro de carbono (Nowak y Crane, 2002; Pimienta de la Torre et al., 2007; Rodríguez-Larramendi et al., 2016).

A diferencia de los bosques, los viñedos tienen un volumen mucho menor de biomasa acumulada, pero bajo un buen manejo agronómico, la tasa anual de secuestro de carbono puede ser alta (Vendrame, 2016; Brunori et al., 2016). Por su condición de plantas leñosas y longevas, los viñedos fijan y retienen parte del bióxido de carbono asimilado para el crecimiento de la madera, por lo que pueden tener una participación importante en el secuestro de carbono atmosférico.

Por lo que, el objetivo de este estudio fue determinar el NEE de un viñedo ( $c v$ Shiraz), su variación a través de los meses de crecimiento y su relación con el secuestro de carbono atmosférico. 


\section{Materiales y métodos}

\section{Ubicación y características del sitio de estudio}

El estudio se realizó durante el ciclo de producción de abril a diciembre de 2018 en un viñedo $c v$ Shiraz de 11 años, propiedad de la Vinícola San Lorenzo, Parras de la Fuente, Coahuila, México $\left(25^{\circ} 26^{\prime}\right.$ latitud norte, $102^{\circ} 10^{\prime}$ longitud oeste, a $\left.1500 \mathrm{msnm}\right)$ con clima seco semicálido, con temperatura media anual de 15 a $20^{\circ} \mathrm{C}$, precipitación promedio anual de $374.2 \mathrm{~mm}$ y tasa de evaporación de $2118 \mathrm{~mm}$ (CONAGUA, 2017). El estudio se realizó en el lote 32 que corresponde a una superficie de 6.37 ha del $c v$ Shiraz, con $1.5 \mathrm{~m}$ de distancia entre plantas y $2.5 \mathrm{~m}$ entre hileras (2 666 planta ha $^{-1}$ ). El viñedo recibió el manejo agronómico (fertilización, poda, riego y control fitosanitario) de acuerdo con los protocolos establecidos por la Vinícola.

\section{Instrumentación y mediciones}

Se usó el método eddy covarianza para medir el NEE entre el dosel del viñedo y la atmosfera de acuerdo con las siguientes ecuaciones (Martens et al., 2004): $\mathrm{NEE}=\mathrm{FCO}_{2}+\frac{\Delta \rho \mathrm{\rho O}_{2}}{\Delta \mathrm{t}} * \Delta \mathrm{z}$ 1), donde $\mathrm{FCO}_{2}=$ es el flujo de $\mathrm{CO}_{2}, \Delta \rho \mathrm{CO}_{2}=$ es el cambio en la densidad del $\mathrm{CO}_{2}$ en un determinado segmento de tiempo $\Delta \mathrm{t}(30 \mathrm{~min})$ y $\Delta \mathrm{z}=$ es la altura ( $3 \mathrm{~m}$ sobre la superficie del suelo) a la que se realizaron las mediciones. El $\mathrm{FCO}_{2}$ se obtuvo con la siguiente relación: $\mathrm{FCO}_{2}=\overline{w^{\prime *} \rho \mathrm{CO}_{2}^{\prime}}$ 2). Donde: $\mathrm{w}=$ es la velocidad vertical del viento, $\mathrm{\rho CO}_{2}=$ es la densidad de bióxido de carbono. Las variables con símbolo de prima significan desviaciones respecto a la media y la barra horizontal sobre dos variables denota la covarianza entre las variables para un determinado segmento de tiempo (30 min).

Los sensores del sistema eddy para realizar las mediciones de los flujos de $\mathrm{CO}_{2}$, se colocaron en un poste a $3 \mathrm{~m}$ de altura (1.2 $\mathrm{m}$ sobre el dosel del viñedo) (Figura 1). La temperatura sónica se midió con un anemómetro sónico tridimensional (CSI-CSAT3, Campbell, Scientific, Inc., Logan, Utah, USA); para obtener $\rho \mathrm{CO}_{2}$ se usó un analizador infrarrojo de bióxido de carbono y vapor de agua de sendero abierto (Open Path $\mathrm{CO}_{2} / \mathrm{H}_{2} \mathrm{O}$ analyzer, LI- 7500. LI-COR, Lincon, Nebraska, USA).

Los sensores se conectaron a un datalogger CR1000 (Campbell, Sci., Inc, Logan, Utah, USA) para realizar mediciones a una frecuencia de $10 \mathrm{~Hz}$ y generar promedios de $30 \mathrm{~min}$. La retención de $\mathrm{CO}_{2}$ del viñedo $\left(\mathrm{mmol} \mathrm{m}^{-2}\right)$ se obtuvo integrando el NEE (promedio de $30 \mathrm{~min}$ ) diurna (valores negativos), mientras que, la tasa de liberación fue la integración de la tasa de NEE nocturna (valores positivos). La retención neta de $\mathrm{CO}_{2}$, fue la diferencia de los valores integrados diurnos y nocturnos.

La tasa de radiación fotosintéticamente activa por sus siglas en inglés (PAR) absorbida se obtuvo colocando dos sensores quánticos (modelo SQ-512, Apogge Inst., Logan, Utah, USA) a un metro sobre el dosel de las plantas, uno orientado hacia el punto medio del dosel y el otro hacia el zénit. La diferencia entre la PAR incidente y la PAR reflejada correspondió a la PAR absorbida por el dosel del viñedo (PAR_abs). Las mediciones se realizaron a una frecuencia de un $\mathrm{Hz}$ y promedios 30 min, conectando los sensores a otro datalogger CR1000. 


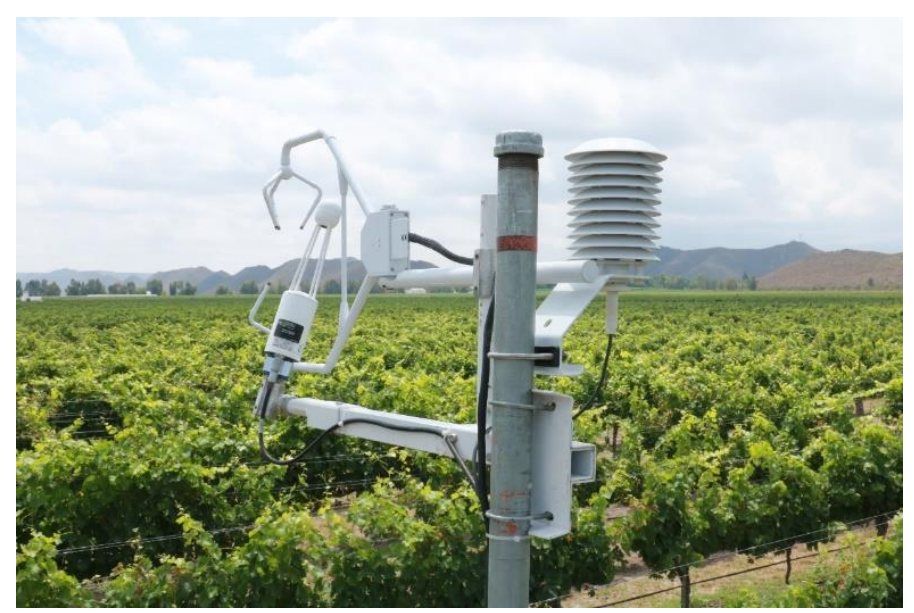

Figura 1. Sensores de un sistema eddy para la medición del flujo de bióxido de carbono entre el dosel del viñedo y la atmósfera. Ciclo de producción 2018. Vinícola San Lorenzo, Parras, Coahuila, México.

El carbono almacenado en las plantas del viñedo se determinó midiendo el diámetro y la longitud del tronco y las ramas de cinco plantas del viñedo (debido a que las plantas son muy uniformes). El volumen promedio de la madera por planta se multiplicó por el número de plantas por hectárea (2 666) para obtener el volumen total de la madera por ha. El peso total de la madera se obtuvo considerando una densidad de madera de $0.701 \mathrm{~g} \mathrm{~cm}^{-3}$ (Nasser et al., 2014). El carbono almacenado se obtuvo asumiendo que $45 \%$ de la composición de la materia seca corresponde a carbono (Yerena-Yamallel et al., 2012).

\section{Resultados y discusión}

\section{Tasa instantánea de intercambio neto de bióxido de carbono}

La máxima tasa instantánea (promedio de $30 \mathrm{~min}$ ) de intercambio neto de bióxido de carbono (NEE) diurno a través de los meses de crecimiento del viñedo (abril a diciembre), se observó a alrededor de las 12:00 h del día (Figuras 2 y 3). En las mismas figuras también se observa que el NEE diurno tiene el mismo patrón de variación que la PAR_abs por el dosel del viñedo, pero con signo contrario (ya que el flujo de $\mathrm{CO}_{2}$ hacia el dosel se establece como negativo). Nótese que la PAR_abs máxima también ocurre a alrededor de las 12:00 h y que es muy sensible a los cambios en las condiciones de nubosidad (Figuras 2 y 3) al igual que el NEE, pero en menor magnitud. Entre abril y agosto ocurrió la tasa instantánea máxima de NEE, donde mayo y junio tuvieron los valores mayores (hasta $-9 \mu \mathrm{mol} \mathrm{m} \mathrm{m}^{-2} \mathrm{~s}^{-1}$ ).

De septiembre a diciembre se tuvo una reducción progresiva de la tasa de NEE (Figura 3). Estudios previos en este cultivo y otras superficies vegetales han mostrado relaciones similares. Por ejemplo, Wofsy et al. (1993) observaron que la tasa de NEE se incrementa sistemáticamente con la incidencia de la PAR en un bosque de latitud media, los valores mayores de NEE también los observaron alrededor de las 12:00 h, y durante el mes de julio el NEE fue mayor. 


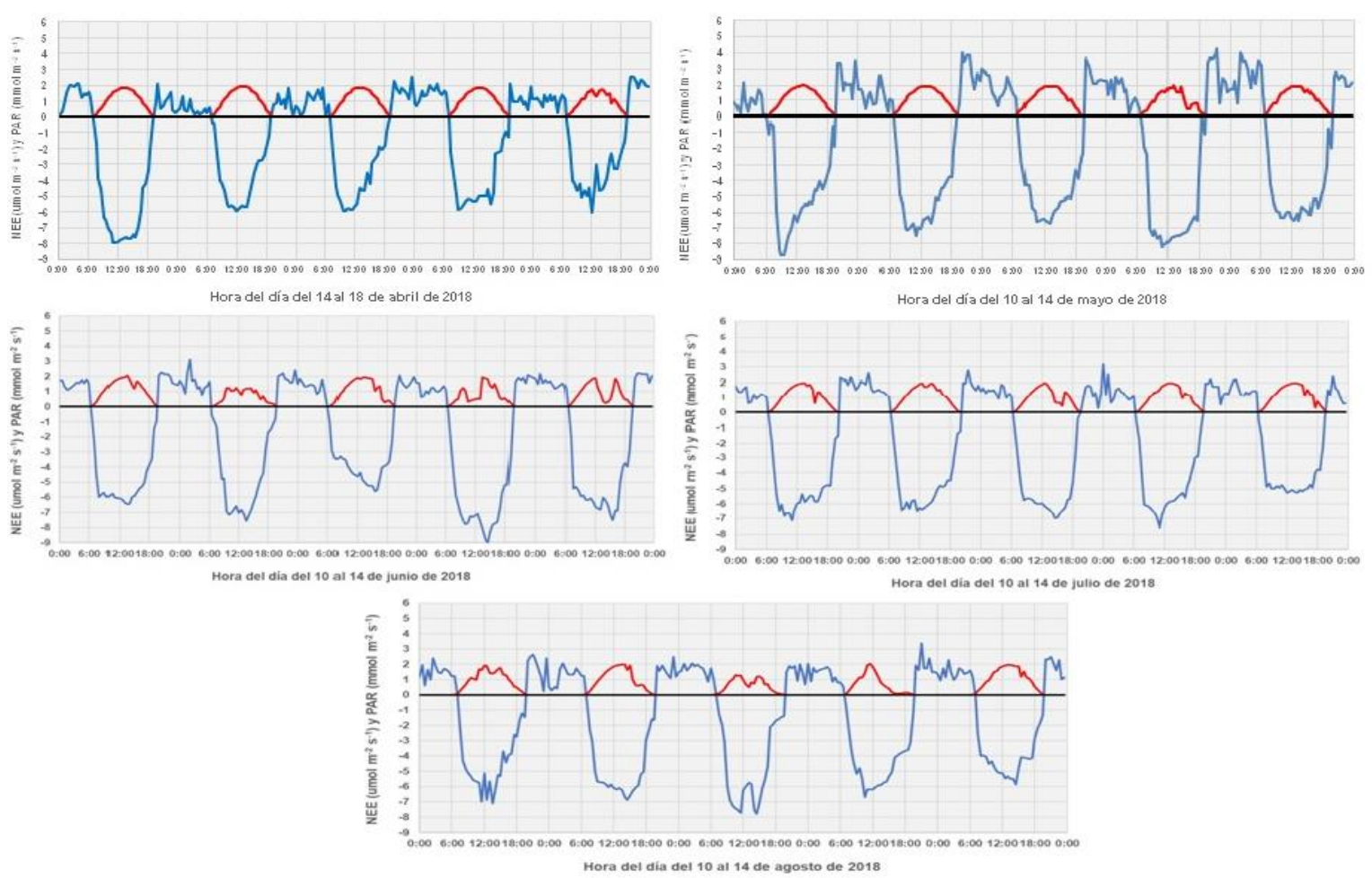

Figura 2. Tasa de intercambio neto de bióxido de carbono (NEE) (líneas de color azul) promedios de 30 min y de asimilación de radiación fotosintéticamente activa (PAR) (líneas de color rojo) absorbida por el dosel del viñedo, durante los meses de abril a agosto de 2018, en la Vinícola San Lorenzo, Parras, Coahuila, México.

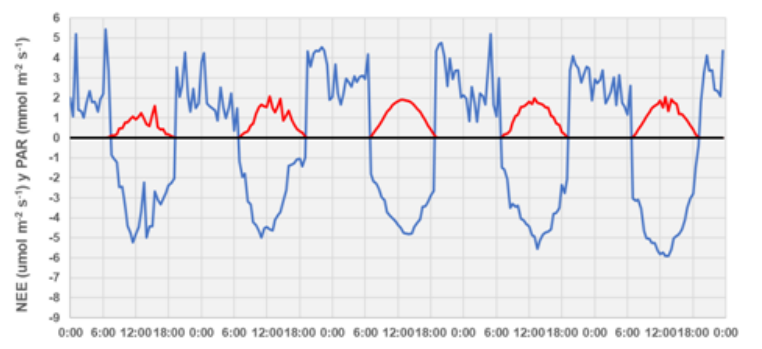

Hora del dia del 10 al 14 de septiembre de 2018

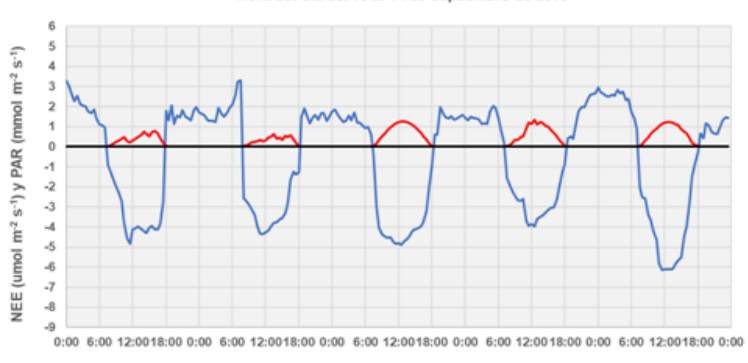

Hora del dia del 12 al 16 de noviembre de 2018
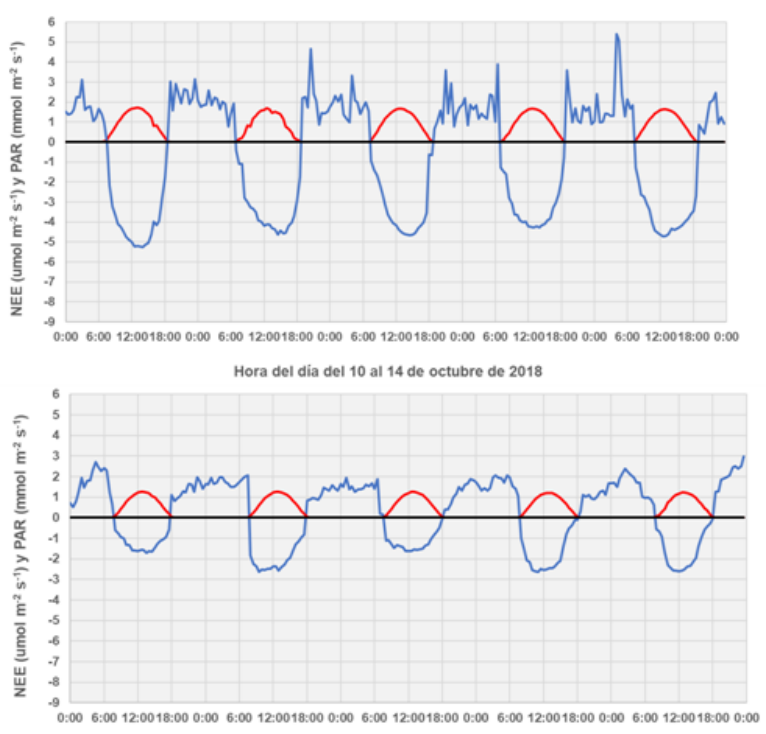

Hora del dia del 1 al 5 de diciembre de 2018

Figura 3. Tasa de intercambio neto de bióxido de carbono (NEE) (líneas de color azul) promedios de 30 min y de asimilación de radiación fotosintéticamente activa (PAR) (líneas de color rojo) absorbida por el dosel del viñedo, durante los meses de septiembre a diciembre de 2018, en la Vinícola San Lorenzo, Parras, Coahuila, México. 
De forma similar a los resultados observados en este estudio, en un viñedo del noroeste árido de China, la tasa horaria de NEE fue positiva (liberación de $\mathrm{CO}_{2}$ ) al inicio del ciclo de producción, valores negativos altos (asimilación) en la etapa media del ciclo de producción y valores negativos pequeños al final del ciclo del viñedo (Guo et al., 2014).

Otros estudios en viñedo de diferentes cultivares y edades, han reportado tasas de NEE similares a las observadas en este estudio. Por ejemplo, en un viñedo de uva de mesa de cultivar Regina, durante el verano en el sur de Italia, la tasa máxima de NEE fue de -5 a $-11 \mu \mathrm{mol} \mathrm{m}{ }^{-2} \mathrm{~s}^{-1}$ (el signo negativo indica asimilación) (Nardino et al., 2007). En un cabernet Sauvignon de 7 años, de California, USA, durante el verano fue de hasta $-13 \mu \mathrm{mol} \mathrm{m} \mathrm{m}^{-2} \mathrm{~s}^{-2}$ (Smart et al., 2009), para el $c v$ Sauvignon Blanc, durante junio en Portogruaro ubicado en el norte de Italia, el NEE fue de hasta $-15 \mu \mathrm{mol} \mathrm{m} \mathrm{m}^{-2} \mathrm{~s}^{-1}$, (Vendrame, 2016).

\section{Tasa integrada diaria de intercambio neto de bióxido de carbono del viñedo}

Los valores de la tasa instantánea (promedio de $30 \mathrm{~min}$ ) de intercambio neto de bióxido de carbono, se integraron por separado para condiciones diurnas (valores negativos) y nocturnas (valores positivos) en los meses de crecimiento del viñedo (abril a diciembre) (Cuadro 1). La diferencia de los valores integrados diurnos y nocturnos representa el carbono que queda fijado para la elaboración de compuestos de carbono (Meggio y Pitacco, 2016; Zanotelli et al., 2018).

Cuadro 1. Promedio diario mensual del intercambio neto de bióxido de carbono (NEE) diurno y nocturno y el balance diario mensual. Vinícola San Lorenzo, ciclo de producción 2018. Parras, Coahuila, México.

\begin{tabular}{cccc}
\hline Mes & $\begin{array}{c}\text { NEE diuron } \\
\left(\mathrm{mmol} \mathrm{m}^{-2} \mathrm{~d}^{-1}\right)\end{array}$ & $\begin{array}{c}\text { NEE nocturno } \\
\left(\mathrm{mmol} \mathrm{m}^{-2} \mathrm{~d}^{-1}\right)\end{array}$ & $\begin{array}{c}\text { NEE neto } \\
\left(\mathrm{mmol} \mathrm{m}^{-2} \mathrm{~d}^{-1}\right)\end{array}$ \\
\hline Abril & -215.647 & 63.882 & -151.765 \\
Mayo & -265.71 & 66.258 & -199.452 \\
Junio & -258.261 & 60.483 & -197.77 \\
Julio & -229.645 & 51.097 & -178.548 \\
Agosto & -195.903 & 68.516 & -127.387 \\
Septiembre & -163.033 & 94.433 & -68.6 \\
Octubre & -151 & 89.214 & -61.786 \\
Noviembre & -120.964 & 73.179 & -47.786 \\
Diciembre & -53.2 & 65.8 & 12.6 \\
\hline
\end{tabular}

De abril a agosto se observó el mayor NEE diurno donde mayo y junio tuvieron los valores más altos (Cuadro 1). De septiembre a diciembre se observó una reducción progresiva de la tasa diurna diaria de NEE; mientras que, de agosto a noviembre se presentaron los valores mayores de NEE nocturno (liberación de $\mathrm{CO}_{2}$ ). El balance neto de NEE promedio diario mensual (diferencia entre el NEE diurno y nocturno) fue mayor de abril a agosto, con valores más altos en mayo y junio (Cuadro 1). 
Los valores menores de balance neto de NEE fueron de septiembre a diciembre con una disminución progresiva. En diciembre el balance neto fue positivo, indicando que el viñedo se comportó como fuente de liberación de $\mathrm{CO}_{2}$ a la atmósfera. El promedio del NEE diurno de los meses de mayo, junio y julio (meses de mayor asimilación) fue $251.205 \mathrm{mmol} \mathrm{m}^{-2} \mathrm{~d}^{-1}$ (Cuadro 1), que corresponde a $-3.014 \mathrm{~g} \mathrm{C} \mathrm{m}^{-2} \mathrm{~d}^{-1}$. Para un viñedo de uva de mesa el NEE promedio diurno fue $-2.079 \mathrm{~g} \mathrm{C} \mathrm{m}^{-2} \mathrm{~d}^{-1}$ (Nardino et al., 2007), mientras que, para un viñedo del cultivar Merlot, el NEE diurno en la etapa del crecimiento de fruto fue hasta $-9 \mathrm{~g} \mathrm{C} \mathrm{m}^{-2} \mathrm{~d}^{-1}$ (Guo et al., 2014).

En otro estudio, Pitacco y Meggio (2016) reportaron que el NEE promedio diario del ciclo de producción de un viñedo del $c v$ Carmenere fue $-2.33 \mathrm{~g} \mathrm{C} \mathrm{m}^{-2} \mathrm{~d}^{-1}$. Los datos anteriores muestran que, los viñedos pueden tener una participación importante en la mitigación del bióxido de carbono atmosférico, por ser plantas leñosas y que pueden estar en producción hasta por 40 años.

\section{Carbono almacenado en el viñedo}

El contenido promedio de carbono por planta del viñedo fue $1.258 \mathrm{~kg}$ (Cuadro 2), con un coeficiente de variación de $11.62 \%$, indicando que las plantas del viñedo son muy uniformes y que la muestra de cinco plantas fue adecuada para la determinación del contenido de carbono en las plantas. Para una densidad de 2666 plantas ha $^{-1}$ equivale a $3.35 \mathrm{t} \mathrm{C} \mathrm{ha}^{-1}$ almacenado en la madera del viñedo. Otros estudios han reportado valores similares a lo encontrado en este estudio.

Cuadro 2. Volumen de madera de cinco plantas de vid ( $c v$ Shiraz) de 11 años, el peso seco y contenido correspondiente de carbono. Vinícola San Lorenzo, Parras, Coahuila.

\begin{tabular}{cccc}
\hline \multirow{2}{*}{ Planta } & $\begin{array}{c}\text { Volumen de la madera } \\
\left(\mathrm{cm}^{3}\right)\end{array}$ & $\begin{array}{c}\text { Peso seco de la madera } \\
(\mathrm{kg})\end{array}$ & $\begin{array}{c}\text { Contenido de carbono } \\
(\mathrm{kg})\end{array}$ \\
\hline 1 & 3821.37 & 2.678 & 1.205 \\
2 & 3575.84 & 2.506 & 1.128 \\
3 & 4464.78 & 3.129 & 1.408 \\
4 & 3578.94 & 2.508 & 1.129 \\
5 & 4505.63 & 3.158 & 1.421 \\
Promedio & 3989.314 & 2.796 & 1.258 \\
\hline
\end{tabular}

Por ejemplo, Williams et al. (2011) señalan que el carbono promedio almacenado en las plantas de cinco cultivares de vid del estado de California, USA fue $3 \mathrm{t} \mathrm{ha}^{-1}$. Estudios realizados en plantas de vid del cultivar Sauvignon en Sacramento California, USA mostraron que el carbono almacenado en la madera es $4.8 \mathrm{t} \mathrm{ha}^{-1}$ (Morandé et al., 2017). En la parte central de Italia en un viñedo del cultivar Merlot, el carbono retenido en la madera fue $2.28 \mathrm{t} \mathrm{ha}^{-1}$ (Brunori et al., 2016).

Estos resultados muestran que, además de la importancia económica y social de los viñedos, también son ecosistemas agrícolas, que tienen una contribución significativa en el secuestro de carbono atmosférico, lo que se debe a su condición de plantas leñosas y longevas y a la superficie establecida tanto en México (32 000 ha) (Boullosa, 2017), como a nivel mundial (7.4 millones de ha) (OIV, 2019). 


\section{Eficiencia quántica del viñedo}

La eficiencia quántica del viñedo (relación entre los milimoles de $\mathrm{CO}_{2}$ asimilados por mol de fotones absorbidos por el dosel) durante los meses del ciclo de producción (abril a diciembre) fue muy similar con muy poca variación entre los diferentes meses (Cuadro 3). El valor menor fue $4.118 \mathrm{mmol} \mathrm{mol}^{-1}$ en agosto y el mayor $5.456 \mathrm{mmol} \mathrm{mol}^{-1}$ en junio. El valor medio de los meses del ciclo de producción fue $4.25 \mathrm{mmol} \mathrm{mol}^{-1}$ con un pequeño coeficiente de variación de $8.99 \%$. La uniformidad de la eficiencia quántica del viñedo es un indicador del buen manejo agronómico del viñedo y la adecuada y uniforme irrigación de las plantas durante los meses de crecimiento.

Cuadro 3. Intercambio neto de bióxido de carbono diurno (NEE diurno), radiación fotosintéticamente active absorbida (PAR_abs) y la eficiencia quántica (Ef quántica) promedio diario mensual del viñedo, durante los meses del ciclo de producción de 2018. Vinícola San Lorenzo, Parras, Coahuila, México.

\begin{tabular}{cccc}
\hline Mes & $\begin{array}{c}\text { NEE diurno } \\
\left(\mathrm{mmol} \mathrm{m}^{-2}\right)\end{array}$ & $\begin{array}{c}\text { PAR_abs } \\
\left(\mathrm{mol} \mathrm{m}^{-2}\right)\end{array}$ & $\begin{array}{c}\text { Ef quántica } \\
\left(\mathrm{mmol} \mathrm{mol}^{-1}\right)\end{array}$ \\
\hline Abril & -215.647 & 47.558 & 4.534 \\
Mayo & -265.71 & 51.499 & 5.16 \\
Junio & -258.261 & 47.333 & 5.456 \\
Julio & -229.645 & 47.159 & 4.87 \\
Agosto & -195.903 & 47.57 & 4.118 \\
Septiembre & -163.033 & 34.306 & 4.752 \\
Octubre & -151 & 32.15 & 4.696 \\
Noviembre & -120.964 & 26.614 & 4.541 \\
Diciembre & -53.2 & 12.6 & 4.222 \\
\hline
\end{tabular}

Los valores de la eficiencia quántica que se observaron en este estudio son menores a los que se reportan en estudios previos, y se debe a que, en este estudio, las mediciones se realizaron a escala dosel con valores integrados diarios (8:00 a 19:00 h), donde se tiene un amplio rango de variaciones de temperatura, humedad y radiación; mientas que, en estudios previos se reportan las eficiencias de mediciones foliares en tiempos cortos (alrededor de un minuto), con condiciones controladas de las variables mencionadas. Por ejemplo, mediciones foliares de la tasa de fotosíntesis de hojas maduras de plantas de vid de los cultivares Riesling y Chasselas a una temperatura de 25 a $30{ }^{\circ} \mathrm{C}$ y una incidencia PAR de $1000 \mu \mathrm{mol} \mathrm{m}{ }^{-2} \mathrm{~s}^{-1}$ fueron $12.66 \mu \mathrm{mol}$ $\mathrm{CO}_{2} \mathrm{~m}^{-2} \mathrm{~s}^{-1}$, que corresponde a un rendimiento quántico de $12.66 \mathrm{mmol} \mathrm{mol}^{-1}$ (Zufferey et al., 2000).

Similarmente, la máxima tasa de fotosíntesis foliar de plantas de vid del $c v$ Semillon, a una temperatura entre 25 y $30{ }^{\circ} \mathrm{C}$ y una PAR de $1000 \mu \mathrm{mol} \mathrm{m} \mathrm{m}^{-2}$ fue $16.25 \mu \mathrm{mol} \mathrm{CO} \mathrm{m}^{-2} \mathrm{~s}^{-1}$, lo que correspondió a una eficiencia quántica de $16.25 \mathrm{mmol} \mathrm{mol}^{-1}$ (Greer y Weedon, 2012). La tasa máxima de fotosíntesis de hojas de vid del $c v$ Chardoney y Merlot para una temperatura de $25{ }^{\circ} \mathrm{C}$, una concentración de Carbono de $400 \mu \mathrm{mol} \mathrm{mol}^{-1}$, una incidencia PAR de $600 \mu \mathrm{mol}$ $\mathrm{m}^{-2} \mathrm{~s}^{-1}$ fue $8.5 \mu \mathrm{mol} \mathrm{CO} \mathrm{m}^{-2} \mathrm{~s}^{-1}$, que correspondió a una eficiencia quántica de $14.16 \mathrm{mmol}$ $\mathrm{mol}^{-1}$ (Greer, 2017). 


\section{Conclusiones}

La máxima NEE (promedios de 30 min), a través de los meses del ciclo de producción (abril a diciembre) se observó alrededor del mediodía y en mayo y junio se presentan los valores más altos. La NEE sigue el mismo patrón de variación que PAR absorbida por el dosel del viñedo. De abril a noviembre la tasa promedio diaria mensual diurna fue mayor que la nocturna, indicando que el viñedo fue sumidero de carbono atmosférico. A partir de diciembre, debido a la senescencia y caída de las hojas, el NEE nocturno fue mayor que el diurno y el viñedo fue fuente de liberación de $\mathrm{CO}_{2}$.

Por el carbono almacenado, la tasa anual de secuestro de carbono y las grandes extensiones de superficie establecida en México y el mundo, los viñedos son sistemas agrícolas de importancia para la asimilación y retención de carbono atmosférico. El rendimiento cuántico promedio diario del viñedo fue muy estable a través de los meses del ciclo de crecimiento del viñedo, que se debe a un buen manejo agronómico del cultivo y la aplicación oportuna del riego.

\section{Agradecimientos}

Los autores agradecen a la Vinícola San Lorenzo por la disposición y apoyo otorgado para la realización del estudio y al Consejo Nacional de Ciencia y Tecnología (CONACYT) por el apoyo de beca (No. 864944) del segundo autor.

\section{Literatura citada}

Boullosa, R. 2017. Producción en Mexico.https://revistaelconocedor.com/produccion-nacional-laindustria-del-vino-mexicano/.

Brunori, E.; Farina, R. and Biasi, R. 2016. Sustainable viticulture: the carbon-sink function of the vineyard agro-ecosystem. Agric. Ecosys. Environ. 223:10-21.

Cano, P. 2015. La vida de la vid. Vínica. http://vinica.es/la-vida-de-la-vid/.

Carrara, A.; Skowalski, A.; Neirynck, J.; Janssens, I. A.; Yuste, J. C. and Ceulemans, R. 2003. Net ecosystem $\mathrm{CO} 2$ exchange of mixed forest in Belgium over 5 years. Agricultural and Forest Meteorology. 119:209-227.

CMV. 2018. Consejo Mexicano Vitivinícola. Línea del tiempo. http://uvayvino.org.mx/index.php/ inicio/linea_tiempo.

CONAGUA. 2017. Comisión Nacional del Agua. Información climatológica por estado. https://smn.conagua.gob.mx/es/informacion-climatologica-por-estado?estado=coah.

Desai, A. R.; Bolstad, P.V.; Cook, B. D.; Davis, K. J. and Carey, E. V. 2005. Comparing net ecosystem exchange of carbon dioxide between an old-growth and mature forest in the upper Midwest, USA. Agricultural and Forest Meteorology. 128:33-55.

Domínguez de la Iglesia, E. 2018. El ciclo vegetativo de la vid. Campus internacional del vino, https://www.campusdelvino.com/blog/item/95-ciclo-vegetativovid\%20dominguez,2018.

El financiero. 2018. Economía, mercados y negocios en alianza. El mercado de vino en México, francamente verde. https://www.elfinanciero.com.mx/bajio/el-mercado-de-vino-enmexico-francamente-verde.

Greer, D. H. and Weedon, M. M. 2012. Modelling photosynthetic responses to temperature (Vitis vinifera $c v$ Semillon) leaves on vines grown in a hot climate. Plant Cell Environ. 35:1050-1064. 
Greer, D. H. 2017. Temperatura and $\mathrm{CO}_{2}$ dependency of the photosynthesis photon flux density response of leaves of Vitis vinifera $c v$. Chardoney and Merlot grown in a hot climate. Plant Physiol. Biochem. 11:295-303.

Guo, W. H.; Kang, S. Z.; Li, F. S. and Li, S. E. 2014. Variations of NEE and its affecting factors in a vineyard of arid region of northwest China. Atmospheric Environment. 84:349-354.

Martens, C. T.; Thomas, J. S.; Mendlovitz, H. P.; Matross, D. M.; Saleska, S. R.; Wofsy, S. C.; Woodward W. S.; Menton, M. C.; De Moura, J. M. S.; Crill, P. M.; De Moraes, O. L. and Lima, R. L. 2004. Radon fluxes in tropical forest ecosystem of brazilian Amazonia: nighttime $\mathrm{CO} 2$ net ecosystem exchange derived from random and eddy covariance methods. Global Change Biol. 10(5):618-629.

Martin-Gorriz, B.; Egea, G.; Nortes, P. A.; Baile, A.; González-Real, M. M.; Ruiz-Salleres, I. and Verhoef A. 2011. Effects of high temperature and vapour pressure deficit on net ecosystem exchange and energy balance of an irrigated orange orchard in a semi-arid climate southern Spain. Acta Hortic. 922:149-156.

Meggio, F. and Pitacco, A. 2016. Carbon budget of a temperate-climate vineyard-a green future for viticulture? Acta Hortic. 1112:455-460.

Morandé, J. A.; Stockert, C. M.; Liles, G. L.; Williams, J. N.; Smart, D. R. and Viers, J. H. 2017. From berries to block: carbon stock quantification of a California vineyard. Carbon Balance and Management. 12(5):1-12.

Nardino, M.; Facini, O.; Georgiadis, T. and Rossi, F. 2007. Canopy observations of a table grape vineyard: radiation balance, energy partitioning and CO2 fluxes. Acta Hortic. 732:611-615.

Nasser, R. A.; Salem, M. Z. M.; Al-Mefarrej, H. A.; Abdel-Aal, M. A. and Soliman, S. S. 2014. Vine pruning for energy. BioResources. 9(1):482-496.

Nowak, D. J. and Crane, D. E. 2002. Carbon storage and sequestration by urban trees in the USA. Environmental Pollution. 116(3):381-389.

OIV. 2019. Organization International of Vine and Wine. La superficie mundial de viñedos se mantiene estable en 2019 según OIV. Agronews Castilla y León. (agronewscastillayleon.com).

Pimienta de la Torre, D. de J.; Domínguez-Cabrera, G.; Aguirre-Calderón, O.; Javier-Hernández, F. y Jiménez-Pérez, J. 2007. Estimación de biomasa y contenido de carbono de Pinus cooperi Blanco, en Pueblo Nuevo, Durango. Madera y Bosques. 13(1):35-46.

Rodríguez-Larramendi, L. A.; Guevara-Hernández, F.; Reyes-Muro, L.; Ovando-Cruz, L.; NahedToral, J.; Prado-López, J. M. y Campos-Saldaña, R. A. 2016. Estimación de biomasa y carbono almacenado en bosques comunitarios de la región Frailesca de Chiapas, México. Rev. Mex. Cienc. Forest. 7(37):77-94.

SADER. 2018. Secretaría de Agricultura y Desarrollo Rural. Fomento a la industria vitivinícola impulso y desarrollo del vino mexicano. https://www.gob.mx/sader/es/articulos/fomentoa-la-industria-vitivinicola-impulso-y-desarrollo-del-vino-mexicano.

Smart, R. D.; Wolff, W. M; Carlisle, E. and María del Mar, A. M. 2009. Reducing greenhouse gas emissions in the vineyard: advances search to develop more sustainable practices. Department of Viticulture \& Enology University of California. 1-13 pp. https://www.academia.edu/2518357/Reducing_Greenhouse_Gas_Emissions_in_the_

Vineyard_Advances_in_the_Search_to_De elop-More_Sustainable_Practices.

Testi, L.; Orgaz, F. and Villalobos, F. 2008. Carbon exchange and water use efficiency of a growing, irrigated olive orchard. Environ. Exp. Bot. 63(1-3):168-177. 
Vendrame, N. 2016. Study of vegetation-atmosphere interactions over vineyards: $\mathrm{CO}_{2}$ fluxes and turbulent transport mechanics. Padova Digital University Archive. 1-16 pp.

Vinetur. 2017. La revista digital del vino. La vida, el origen del vino. https:/www.vinetur.com/ 2017032827627/la-vid-el-origen-del-vino.html.

Williams, J. N.; Hollander, A. D.; O’Green, A. T.; Thrupp, L. A.; Hanifin, R.; Steenwerth, K.; McGourty G. and Jackson, L. E. 2011. Assessment of carbon in woody plants and soil across a vineyard-woodland landscape. Carbob Balance and Management. 6(11):1-14.

Wofsy, S. C.; Goulden, M. L.; Munger, J.W.; Fan, S. M., Bakwin, P. S.; Daube, B. C. and Bazzaz, F. A. 1993. Net Exchange of CO2 in a Mid-Latitude Forest Science. 260(5112):1314-1317.

Yerena-Yamallel, J. I.; Jiménez-Pérez, J.; Aguirre-Calderón, O. A. y Treviño-Garza, E. J. 2012. Contenido de carbono total en los componentes de especies arbóreas y arbustivas en áreas con diferente uso, en el matorral espinoso tamaulipeco, en México. Bosque. 33(2):145-152.

Yi, C.; Anderson, D. E.; Turnipseed, A. A.; Burns, S. P.; Sparks, J. P.; Stannard, D. I. and Monson, R. K. 2008. The contribution of advective fluxes to net ecosystem exchange in a high elevation, subalpine forest. Ecological Applications. 18(6):1379-1390.

Zanotelli, D.; Montagnani, L.; Manca, G. and Tagliavini, M. 2013. Net primary productivity, allocation pattern and carbon use efficiency in an apple orchard assessed by integrating eddy covariance, biometric and continuous soil chamber measurements. Biogeosciences. 10(5):3089-3108.

Zanotelli, D.; Vendrame, N.; López-Berna, A. and Caruso, G. 2018. Carbon sequestration in orchards and vineyards. Italus Hortus. 25(3):13-28.

Zufferey, V.; Murisier, F. and Schultz, H. R. 2000. A model analysis of the photosynthetic response of Vitis vinifera L. $c v$ Riesling and Chasselas leaves in the field: I. Interaction of age, light and temperature. Vitis. 39(1):19-26. 